\title{
Produtividade de alho vernalizado em função de fontes e doses de fósforo
}

\author{
Productivity of vernalized garlic in function of sources and doses of phosphorous
}

\author{
Fábio Silva MacêdoI Ériko Tadashi Sedoguchi" Rovilson José de Souza ${ }^{\text {III }}$ \\ Janice Guedes de Carvalho ${ }^{\mathrm{III}}$
}

\section{RESUMO}

\begin{abstract}
Objetivou-se com este trabalho verificar a influência de fontes e doses de fósforo na produtividade do alho vernalizado ( $\mathrm{cv}$. 'Roxo Pérola de Caçador') em solo com baixo teor de fósforo. $O$ experimento foi conduzido em condições de campo no Setor de Olericultura da Universidade Federal de Lavras. Foram avaliadas três fontes de P (superfosfato simples, com $18 \%$ de $\mathrm{P}_{2} \mathrm{O}_{5}$; superfosfato triplo, com $41 \%$ de $\mathrm{P}_{2} \mathrm{O}_{5}$ e termofosfato magnesiano, com $17 \%$ de $\mathrm{P}_{2} \mathrm{O}_{5}$ ), três doses $\left(200,400\right.$ e $600 \mathrm{~kg} \mathrm{ha}^{-1}$ de $\left.\mathrm{P}_{2} \mathrm{O}_{5}\right)$ e um tratamento adicional, que não recebeu adubação fosfatada. A aplicação de $200 \mathrm{~kg}$ ha-l de $\mathrm{P}_{2} \mathrm{O}_{5}$, utilizando como fonte o superfosfato triplo, proporcionou maior ganho em fitomassa total de plantas e melhores resultados com relação à produtividade de bulbos comerciais.
\end{abstract}

Palavras-chave: Allium sativum, frigorificação, nutrição mineral.

\section{ABSTRACT}

The objective of this research was to verify the influence of sources and doses of phosphorus in the productivity of vernalized garlic in soil with low phosphorus content. The experiment was carried out in field conditions in the Horticulture Sector at the Universidade Federal de Lavras. Three sources (simple superphosphate, with $18 \%$ of $\mathrm{P}_{2} \mathrm{O}_{5}$; triple superphosphate, with $41 \%$ of $\mathrm{P}_{2} \mathrm{O}_{5}$ and magnesium term phosphate, with $17 \%$ of $\left.\mathrm{P}_{2} \mathrm{O}_{5}\right)$, three doses $(200,400 \mathrm{e} 600 \mathrm{~kg}$ ha $a^{-1}$ de $P_{2} O_{5}$ ), and one additional treatment, that didn't receive manuring with phosphorus were tested. The application of $200 \mathrm{~kg} \mathrm{ha}^{-1}$ of $\mathrm{P}_{2} \mathrm{O}_{5}$, using triple superphosphate provides the best profit in total mass of plants and productivity of commercial bulbs.

Key words: Allium sativum, vernalization, mineral nutrition.

\section{INTRODUÇÃO}

Dentre as espécies iônicas originadas do ácido ortofosfórico $\left(\mathrm{H}_{3} \mathrm{PO}_{4}\right)$, predomina, na faixa de $\mathrm{pH}$ do solo de 4 a 7 , a forma de fosfato $\left(\mathrm{H}_{2} \mathrm{PO}_{4}^{-}\right)$, que é absorvida pelas raízes das plantas. Geralmente, a concentração de fosfato nas células radiculares e na seiva do xilema é de 100 a 1.000 vezes maior do que a da solução do solo. Isso demonstra que a absorção de fosfato dá-se contra um alto gradiente de concentração, portanto, ele é absorvido ativamente (FAQUIN, 2001).

As quantidades de fósforo $(\mathrm{P})$ exigidas pelas culturas são geralmente baixas, principalmente quando comparadas com o nitrogênio e o potássio. Entretanto, apesar dessa baixa exigência, os teores desse nutriente, bem como a velocidade do seu restabelecimento na solução do solo, não são suficientes para atender às necessidades das culturas. Como consequência desses fatos, nas adubações, é o fósforo o nutriente que entra em maiores proporções (COUTINHO et al., 1993). No Brasil, as aplicações de fósforo são realizadas em altas proporções na cultura do alho, principalmente por causa da fixação de grande parte dos fertilizantes fosfatados solúveis (BÜLL et al., 2004).

No alho, a deficiência de fósforo causa redução no crescimento e amarelecimento irregular das folhas mais velhas, progredindo do ápice em direção à base, podendo ser confundida com sintoma de deficiência de nitrogênio (MAGALHÃES, 1986).

'Empresa Brasileira de Pesquisa Agropecuária (EMBRAPA), Brasília, DF, Brasil.

IDepartamento de Sanidade Vegetal, Secretaria de Defesa Agropecuária, Ministério da Agricultura, Pecuária e Abastecimento (MAPA), 70043-900, Brasília, DF, Brasil. E-mail: eriko.sedoguchi@agricultura.gov.br. Autor para correspondência.

"I'Universidade Federal de Lavras (UFLA), Lavras, MG, Brasil. 
Maiores doses de fósforo no solo podem reduzir a absorção de outros nutrientes como $\mathrm{Zn}, \mathrm{Cu}$ e $\mathrm{Fe}$, por causa da precipitação destes, podendo, assim, interferir no crescimento das culturas (VIDIGAL et al., 2002).

Durante a fase de máximo desenvolvimento da parte aérea e do bulbo, ocorrem diferenças no acúmulo de nutrientes entre as plantas propagadas de forma convencional e com o uso de cultura de tecidos. As plantas obtidas por cultura de tecidos acumulam quantidade significativamente maior de nutrientes (RESENDE et al., 1999).

Os efeitos dos períodos de frigorificação, a $15^{\circ} \mathrm{C} \mathrm{e} 4^{\circ} \mathrm{C}$, na superação da dormência, na vernalização e na produtividade de alho da cultivar 'Roxo Pérola de Caçador' foram estudados por REGHIN \& KIMOTO (1998). Para planejamento do plantio em abril, foram recomendados períodos mais curtos de frigorificação, como dez dias a $15^{\circ} \mathrm{C}$ e quarenta dias a $4^{\circ} \mathrm{C}$, realizado imediatamente antes do plantio.

No caso de alho vernalizado, as pesquisas sobre a adubação fosfatada ainda são escassas. Não se chegou ainda a um consenso com relação às quantidades e fontes de fósforo que proporcionam maiores ganhos em produtividade. $\mathrm{O}$ que se tem observado é que a resposta à adubação fosfatada depende em grande parte do tipo e da fertilidade do solo. Em alguns casos, mesmo solos com baixos teores de fósforo, não têm respondido à aplicação do nutriente.

Ao estudarem os efeitos de quatro doses $\left(50,100,200\right.$ e 400mg P dm$\left.{ }^{-3}\right)$ e modos de aplicação de fósforo em cultivos de alho vernalizado, BÜLL et al. (2004) observaram que a produção de bulbos aumentou com as doses de fósforo e foi influenciada pela localização do fertilizante fosfatado apenas no solo argiloso.

O objetivo deste trabalho foi verificar a influência de diferentes fontes e doses de fósforo na produtividade do alho vernalizado.

\section{MATERIAL E MÉTODOS}

O experimento foi conduzido em condições de campo no setor de Olericultura da Universidade Federal de Lavras, no período de 26/04/2006 à 16/09/ 2006. O solo da área foi classificado como Latossolo Vermelho distroférrico e, de acordo com o resultado da análise de solo apresentado na tabela 1, apresentava baixo teor de fósforo. Foram utilizadas três fontes de fósforo (superfosfato simples, com $18 \%$ de $\mathrm{P}_{2} \mathrm{O}_{5}$; superfosfato triplo, com $41 \%$ de $\mathrm{P}_{2} \mathrm{O}_{5}$ e termofosfato magnesiano, com $17 \%$ de $\mathrm{P}_{2} \mathrm{O}_{5}$ ), com três diferentes doses $\left(200,400\right.$ e $600 \mathrm{~kg} \mathrm{ha}^{-1}$ de $\left._{2} \mathrm{O}_{5}\right)$ e um tratamento adicional, que não recebeu adubação fosfatada, caracterizando tratamentos fatoriais no formato $3 \times 3+1$.

Além das quantidades totais dos fertilizantes fosfatados, foram aplicados na adubação de plantio $35 \mathrm{~kg} \mathrm{ha}^{-1}$ de $\mathrm{N}, 200 \mathrm{~kg} \mathrm{ha}^{-1}$ de $\mathrm{K}_{2} \mathrm{O}$ e $3 \mathrm{~kg} \mathrm{ha}^{-1}$ de B, utilizando-se como fontes uréia, cloreto de potássio e bórax, respectivamente. Os fertilizantes foram incorporados nos canteiros dez dias antes do plantio.

Tabela 1 - Resultados das análises física e química do solo da área experimental*.

\begin{tabular}{llll}
\hline Sigla & \multicolumn{1}{c}{ Descrição } & Unidade & Valor \\
\hline $\mathrm{pH}$ & Em água (1:2,5) & - & 5,8 \\
$\mathrm{P}$ & Fósforo (Mehlich) 1) & $\mathrm{mg} \mathrm{dm}^{-3}$ & 4,3 \\
$\mathrm{~K}$ & Potássio & $\mathrm{mg} \mathrm{dm}^{-3}$ & 48 \\
$\mathrm{Ca}$ & Cálcio & $\mathrm{cmol}_{\mathrm{c}} \mathrm{dm}^{-3}$ & 3,2 \\
$\mathrm{Mg}$ & Magnésio & $\mathrm{cmol}_{\mathrm{c}} \mathrm{dm}^{-3}$ & 0,9 \\
$\mathrm{Al}$ & Alumínio & $\mathrm{cmol}_{\mathrm{c}} \mathrm{dm}^{-3}$ & 0,0 \\
$\mathrm{H}+\mathrm{Al}$ & Ac. potencial & $\mathrm{cmol}_{\mathrm{c}} \mathrm{dm}^{-3}$ & 2,9 \\
$\mathrm{SB}$ & Soma de bases & $\mathrm{cmol}_{\mathrm{c}} \mathrm{dm}^{-3}$ & 4,2 \\
(t) & CTC efetiva & $\mathrm{cmol}_{\mathrm{c}} \mathrm{dm}^{-3}$ & 4,2 \\
$(\mathrm{~T})$ & CTC a pH 7,0 & $\mathrm{cmol}_{\mathrm{c}} \mathrm{dm}^{-3}$ & 7,1 \\
$\mathrm{~V}$ & Sat. bases & $\%$ & 59,3 \\
$\mathrm{~m}$ & Sat. alumínio & $\%$ & 0 \\
MO & Mat. orgânica & $\mathrm{dag} \mathrm{kg}^{-1}$ & 2,9 \\
P-rem & Fósforo remanescente & $\mathrm{mg} \mathrm{L}^{-1}$ & 7,5 \\
Areia & - & $\mathrm{dag} \mathrm{kg}^{-1}$ & 16 \\
Silte & - & $\operatorname{dag~kg}^{-1}$ & 22 \\
Argila & - & dag kg & 62 \\
Textura & Classe textural & - & Muito Argilosa \\
\hline
\end{tabular}

*Realizadas no DCS/UFLA. 
Em cobertura, foram aplicados $20 \mathrm{~kg} \mathrm{ha}^{-1}$ de $\mathrm{N}$ aos 55 dias e $40 \mathrm{~kg} \mathrm{ha}^{-1} \mathrm{de} \mathrm{N}$ aos 90 dias, na forma de ureia.

A cultivar utilizada foi a 'Roxo Pérola de Caçador', sendo os bulbos-semente submetidos a um período de vernalização de 50 dias, em câmara com temperatura média de $4^{\circ} \mathrm{C}$. Aos 60 dias após o plantio, a irrigação foi suspensa por 16 dias, visando a diminuir a incidência de superbrotamento. A irrigação também foi suspensa dez dias antes da colheita.

A colheita foi efetuada aos 137 dias após o plantio. Em seguida, as plantas foram secas ao sol por três dias e curadas à sombra por 60 dias. Após a cura, foi efetuado o toalete dos bulbos, sendo então anotados os dados de produção. Foram avaliadas a fitomassa total das plantas $\left(\mathrm{g} .0,6 \mathrm{~m}^{-2}\right)$, a produtividade dos bulbos comerciais (g.0,6 $\mathrm{m}^{-2}$ ), a fitomassa média de bulbilhos da produção comercial (g) e a porcentagem de superbrotamento em função das fontes e doses de fósforo estudadas.

Após a cura, foi obtida a fitomassa total das plantas colhidas em cada parcela. A produtividade dos bulbos comerciais foi obtida considerando-se bulbos com diâmetro acima de $32 \mathrm{~mm}$, não superbrotados e sem chochamento. Por meio do quociente entre fitomassa e número de bulbilhos de uma amostra de dez bulbos comerciais, obteve-se a fitomassa média de bulbilhos. A porcentagem da ocorrência de superbrotamento foi determinada pela contagem dos bulbos superbrotados em relação ao número total colhido em cada um dos tratamentos. Os dados de porcentagem de superbrotamento foram transformados para arco seno $\sqrt{x / 100}$, para a realização da análise de variância.

O delineamento usado foi o de blocos casualizados, com três repetições. As parcelas foram dispostas em canteiros e foram compostas por cinco linhas de plantio, cada uma com $2 \mathrm{~m}$ de comprimento, espaçadas em $0,2 \mathrm{~m}$. O espaçamento entre plantas adotado foi de $0,10 \mathrm{~m}$. A área útil foi definida pelas três fileiras centrais, subtraindo-se ainda $0,5 \mathrm{~m}$ nas extremidades cada linha.

Os dados foram submetidos à análise de variância, com realização do teste $F$, e as comparações entre os tratamentos fatoriais foram efetuadas por meio do teste de Dunnett e análises de regressão, a 5\% de probabilidade. As análises estatísticas foram efetuadas com auxílio do sistema Sisvar.

\section{RESULTADOS E DISCUSSÃO}

Houve diferença significativa da fitomassa total das plantas em função de fontes e doses de fósforo. Os valores obtidos reduziram linearmente com o aumento nas doses de fósforo avaliadas quando foram utilizados o superfosfato simples e o superfosfato triplo (Figura 1).

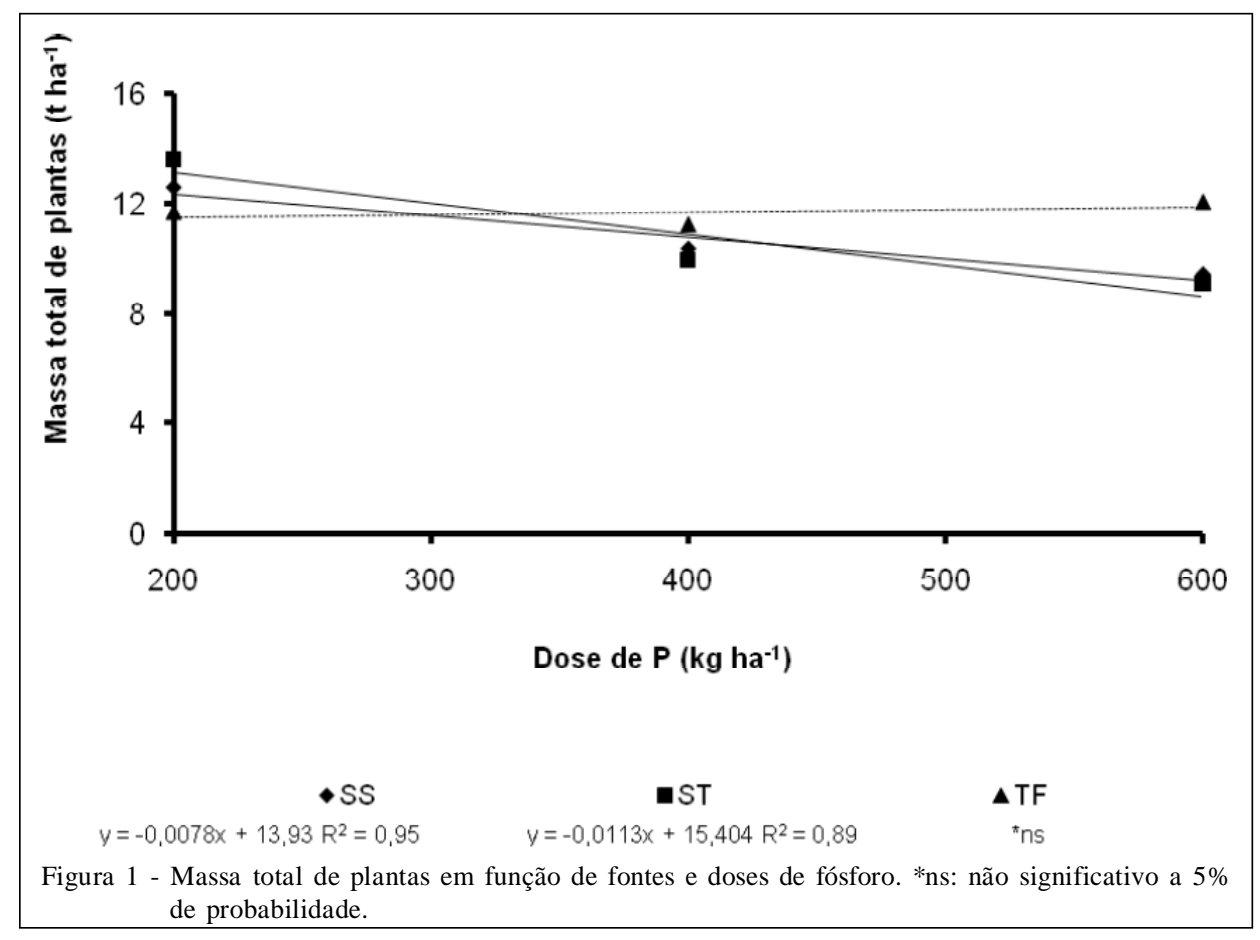

Ciência Rural, v.41, n.3, mar, 2011. 
Maiores doses de fósforo no solo podem reduzir a absorção de outros nutrientes como $\mathrm{Zn}, \mathrm{Cu}$ e $\mathrm{Fe}$, por causa da precipitação destes, podendo assim interferir no crescimento das culturas (VIDIGAL et al., 2002). É interessante ressaltar que experimentos conduzidos por BÜLL et al. (1998, 2004 e 2008) não apresentaram efeitos antagônicos do $\mathrm{P}$ sobre a absorção de $\mathrm{Zn}$, em casa de vegetação.

No caso do termofosfato, não foram observadas diferenças significativas entre as doses avaliadas para nenhuma das características produtivas. Provavelmente, o termofosfato, por ser uma fonte de baixa solubilidade em água, disponibilizou menor quantidade de fósforo ao longo do ciclo da cultura em relação aos superfosfatos, que são fontes de alta solubilidade.

Comparando-se os tratamentos componentes do fatorial com a testemunha, constatou-se que a fitomassa total de plantas obtida na testemunha foi inferior apenas à observada no tratamento com $200 \mathrm{~kg} \mathrm{ha}^{-1}$ de $\mathrm{P}_{2} \mathrm{O}_{5}$, utilizando-se como fonte o superfosfato triplo (Tabela 2).

A produtividade dos bulbos comerciais foi proporcional à fitomassa total das plantas, de forma que a análise de variância apresentou resultados semelhantes, com diferenças significativas em função de fontes e doses de fósforo. Houve redução linear da produtividade com o aumento nas doses de fósforo avaliadas, quando foram utilizados o superfosfato

Tabela 2 - Fitomassa total de plantas (MTP) e produtividade dos bulbos comerciais (PBC) em função de fontes e doses de fósforo.

\begin{tabular}{|c|c|c|c|}
\hline \multicolumn{4}{|c|}{ Tratamentos } \\
\hline Fontes & $\begin{array}{c}\text { Doses } \\
\left(\mathrm{kg} \mathrm{ha}^{-1}\right)\end{array}$ & $\operatorname{MTP}\left(\right.$ g. $\left.0,6 \mathrm{~m}^{-2}\right)$ & PBC (g. $\left.0,6 \mathrm{~m}^{-2}\right)$ \\
\hline $\mathrm{SS}^{1}$ & 200 & $943,67^{\text {ns }}$ & $673,33 *$ \\
\hline SS & 400 & $777,68^{\text {ns }}$ & $458,33^{\text {ns }}$ \\
\hline SS & 600 & $709,00^{\text {ns }}$ & $427,33^{\mathrm{ns}}$ \\
\hline $\mathrm{ST}^{2}$ & 200 & $1021,33^{*}$ & $727,33 *$ \\
\hline ST & 400 & $747,33^{\text {ns }}$ & $391,00^{\mathrm{ns}}$ \\
\hline ST & 600 & $683,33^{\text {ns }}$ & $398,67^{\mathrm{ns}}$ \\
\hline $\mathrm{TF}^{3}$ & 200 & $878,33^{\text {ns }}$ & $537,33^{\text {ns }}$ \\
\hline $\mathrm{TF}$ & 400 & $844,00^{\mathrm{ns}}$ & $480,67^{\mathrm{ns}}$ \\
\hline $\mathrm{TF}$ & 600 & $905,67^{\mathrm{ns}}$ & $606,00^{\mathrm{ns}}$ \\
\hline \multicolumn{2}{|c|}{ Controle } & 823,33 & 462,00 \\
\hline
\end{tabular}

* significativo em relação ao controle, a 5\% de probabilidade pelo teste de Dunnett.

${ }^{\text {ns }}$ não significativo.

${ }^{1}$ superfosfato simples.

${ }^{2}$ superfosfato triplo.

${ }^{3}$ termofosfato magnesiano. simples e o superfosfato triplo (Figura 2). Os tratamentos com $200 \mathrm{~kg} \mathrm{ha}^{-1} \mathrm{de}_{2} \mathrm{O}_{5}$, utilizando-se como fonte superfosfato simples e superfosfato triplo, apresentaram valores superiores aos observados no tratamento testemunha (Tabela 2).

A fitomassa média de bulbilhos, não apresentou diferenças significativas entre os tratamentos componentes do fatorial. Dessa forma, a influência do fósforo na produtividade dos bulbos comerciais parece estar relacionada à formação de maior número de bulbilhos.

Verificou-se que a aplicação de $\mathrm{P}_{2} \mathrm{O}_{5}$, nas fontes e doses estudadas, não exerceu influência sobre a porcentagem de superbrotamento. Todos os tratamentos apresentaram resultados semelhantes aos do tratamento controle. Na literatura, não foram verificadas referências ao fósforo, relacionando-o com tal anormalidade, o que está de acordo com os resultados do presente trabalho.

A suspensão da irrigação por 16 dias, durante a condução do experimento, aliada à uma adubação racional com $\mathrm{N}$, evitando o excesso do elemento, certamente exerceram influência sobre a ocorrência do superbrotamento.

Em regiões produtoras de alho vernalizado, no Estado de Minas Gerais, que adotam alta tecnologia, os alicultores vêm usando, no plantio, $600 \mathrm{a} 750 \mathrm{~kg} \mathrm{ha}^{-1}$ de $\mathrm{P}_{2} \mathrm{O}_{5}$. Os resultados obtidos sugerem que esses agricultores estão utilizando quantidades acima da que seria recomendável técnica e economicamente. Considerando os altos custos dos adubos, pesquisas nessas regiões são de elevada importância para avaliar melhor o uso do fósforo na cultura do alho.

\section{CONCLUSÃO}

A aplicação de $200 \mathrm{~kg} \mathrm{ha}^{-1}$ de $\mathrm{P}_{2} \mathrm{O}_{5}$, utilizando como fonte o superfosfato triplo, proporcionou maior ganho em fitomassa total de plantas. Esse tratamento também proporcionou melhores resultados com relação à produtividade de bulbos comerciais $\left(12,12 \mathrm{t} \mathrm{ha}^{-1}\right)$. Nenhuma das fontes e doses estudadas exerceu influência sobre a fitomassa média dos bulbilhos e a porcentagem de superbrotamento.

\section{AGRADECIMENTO}

Ao Departamento de Agricultura da Universidade Federal de Lavras (UFLA), por disponibilizar a área para instalação do experimento. 


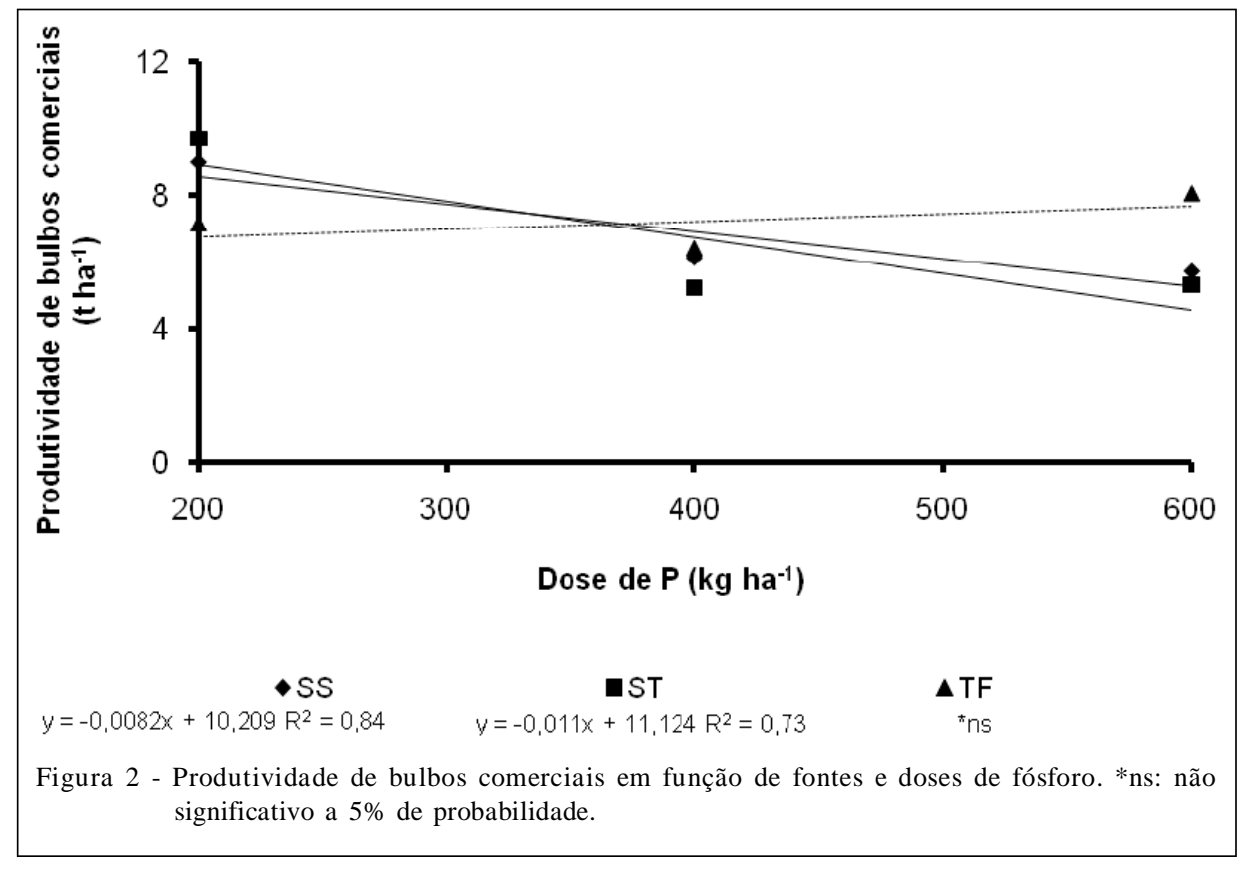

\section{REFERÊNCIAS}

BÜLL L.T. et al. Relações entre fósforo extraído por resina e respostas da cultura do alho vernalizado a adubação fosfatada em cinco solos com e sem adubação orgânica. Revista Brasileira de Ciência do Solo, Viçosa, v.22, p.458-470, 1998.

BÜLL L.T. et al. Doses and forms of application of phosphorus in vernalized garlic. Scientia Agrícola, Piracicaba, v.61, n.5, p.516-521, 2004. Disponível em: <http://www.scielo.br/ scielo.php? script=sci_arttext\&pid=S 0103 90162004000500009>. Acesso em: 29 jul. 2008. doi: 10.1590/ S0103-90162004000500009.

BÜLL L.T. et al. Doses de fósforo e zinco na cultura do alho em condições de casa de vegetação. Bragantia, Campinas, v.67, n.4, p.941-949, 2008. Disponível em: <http:// www.scielo.br/scielo.php? script $=$ sci_arttext \&pid=S000687052008000400017>. Acesso em: 01 jan. 2010. doi: 10.1590/S0006-87052008000400017.

COUTINHO E.L.M. et al. Adubos e corretivos: aspectos particulares na olericultura. In: FERREIRA M.E. et al. (Eds.).
Nutrição e adubação de hortaliças. Piracicaba: Potafos, 1993. p.85-140.

FAQUIN, V. Nutrição mineral de plantas. Lavras: UFLA/ FAEPE, 2001. 182p.

MAGALHÃES, JR. Nutrição mineral do alho. Informe Agropecuário, Belo Horizonte, v.12, p.20-30, 1986.

REGHIN, M.Y.; KIMOTO, T. Dormência, vernalização e produção de alho após diferentes tratamentos de frigorificação de bulbilhos-semente. Horticultura Brasileira, Brasília, v.16, n.1, p. 73-79, 1998.

RESENDE, F.V. et al. Comparação do crescimento e produção entre alho proveniente de cultura de tecidos e de multiplicação convencional. Horticultura Brasileira, Brasília, v.17, n.2, p.118-124, 1999.

VIDIGAL, S.M. et al. Nutrição mineral e adubação da cebola. Informe Agropecuário, Belo Horizonte, v.23, n.218, p.3650, 2002. 